\title{
Sistema ERP para el Seguimiento de Órdenes de Producción
}

\section{ERP System for Tracking Production Orders}

\author{
LEDESMA-URIBE, Norma Alejandra $\dagger$, LEDESMA-GARFIAS, Arisbeth y OLIVO-FLORES, Marco \\ Antonio
}

Universidad Tecnológica de San Juan del Río

ID $1^{\text {er }}$ Autor: Norma Alejandra, Ledesma-Uribe / ORC ID: 0000-0001-8422-2046, Researcher ID Thomson: S-48332018, CVU CONACYT ID: 673202

ID $1^{\text {er }}$ Coautor: Arisbeth, Ledesma-Garfias / ORC ID: 0000-0003-0569-7911, Researcher ID Thomson: Z-4911-2019, CVU CONACYT ID: 997042

ID $2^{\text {do }}$ Coautor: Marco Antonio, Olivo-Flores / ORC ID: 0000-0002-8165-5062, Researcher ID Thomson: S-4865-2018, CVU CONACYT ID: 585138

DOI: $10.35429 / J E D .2019 .18 .6 .9 .16$

Recibido: 20 de enero de 2019; Aceptado: 20 de mayo de 2019

\begin{abstract}
Resumen
En un mundo empresarial cada vez más competitivo, vivimos con la necesidad constante de agilizar, mejorar y potencializar la productividad de nuestros recursos y nuestra organización, para convertir nuestra información en conocimiento inteligente. Para esto, es ideal poder contar con esta información en línea, en tiempo real, desde cualquier dispositivo y desde cualquier lugar del mundo. Estas condiciones permiten tomar decisiones asertivas de manera mucho más ágil. El desarrollo del ERP para el control de seguimiento de órdenes en la empre Zetrak S.A de C.V. tiene como finalidad automatizar los procesos productivos por medio del acceso a la información de manera confiable, precisa y oportuna, dando como resultado la reducción de tiempos y costos en cada uno de los procesos que involucran las operaciones de producción y de distribución de la compañía. Se utilizó como metodología de desarrollo el Modelo en Espiral, que permite producir versiones cada vez más completas durante el desarrollo del sistema, hasta generar la iteración final. El sistema está conformado en su primera etapa por los módulos: Embarques, Ingeniería, Ventas, Facturación y Control de Calidad.
\end{abstract}

ERP, Base de datos, Servidor, Php, Html, módulo, metodología

\begin{abstract}
In an increasingly competitive business world, we live with the constant need to speed up, improve and potentiate the productivity of our resources and our organization, to turn our information into intelligent knowledge. For this, it is ideal to have this information online, in real time, from any device and from anywhere in the world. These conditions allow to make assertive decisions in a much more agile way. The development of the ERP for the control of order tracking in the company Zetrak S.A de C.V. Its purpose is to automate production processes through access to information in a reliable, accurate and timely manner, resulting in the reduction of time and costs in each of the processes involved in the production and distribution operations of the company. The Spiral Model was used as a development methodology, which allows producing more and more complete versions during the development of the system, until generating the final iteration. The system is formed in its first stage by the modules: Shipments, Engineering, Sales, Billing and Quality Control.
\end{abstract}

ERP, Database, Server, Php, Html, module, methodology

Citación: LEDESMA-URIBE, Norma Alejandra, LEDESMA-GARFIAS, Arisbeth y OLIVO-FLORES, Marco Antonio. Sistema ERP para el Seguimiento de Órdenes de Producción. Revista de Desarrollo Económico. 2019, 6-18. 9-16

\footnotetext{
$\dagger$ Investigador contribuyendo como primer autor.
} 


\section{Introducción}

Los sistemas ERP están diseñados para adaptarse a los diferentes tipos de negocios. Normalmente un ERP de última generación, admite con su versión estándar modelar los procesos de cualquier tipo de negocio, gracias a su robusta parametrización y configuración. Así el sistema se podrá adaptar a las necesidades de su empresa y no su empresa al ERP. El proyecto de implementación del ERP tiene una gran importancia para que esto suceda, por lo tanto, el equipo implementador debe ser de una alta calidad humana y competitiva. (Cuetter, 2017)

Un Sistema ERP es un conjunto de aplicaciones de software que permite la integración de las operaciones de una empresa. Dichas operaciones pueden ser Capital Humano, desarrollo de proveedores, compras, ventas, almacenes, producción y embarques, dando la seguridad y comodidad de unificación en el sistema(TIC portal, 2019). La gran aceptación de los sistemas ERP's radican en lo versátiles que pueden ser, ya que los prcesos de negocio son definidos por el tipo de organización en la que se vaya a utilizar. Su diseño permite un flujo de información tan preciso, como el mismo flujo de la materia prima desde que es solicitada hasta que es llevada a la línea de producción, transformada en producto terminado y este sale de la empresa rumbo al cliente.

Estos procesos permiten que integrar los datos de las transacciones originados $y$ modificados en alguno de las áreas de la organización, se evite la duplicidad, y a su vez, permita la actualización de la información disponible y segura en todo momento como "fuente de verdad". (Oracle, 2019)

Las principales funciones de un ERP son:

$\begin{array}{ll}\text { - } & \text { La gestión documental (DMS) } \\ \text { - } & \text { Gestión de pedidos } \\ \text { - } & \text { Gestión de facturación } \\ \text { - } & \begin{array}{l}\text { Planificación de la cadena de } \\ \text { producción y entrega }\end{array} \\ \text { - Gestión de inventarios y almacenes } \\ \text { (WMS) } \\ \text { - Gestión de ciclo de vida del producto } \\ \text { - } \quad \text { Gestión de la calidad }\end{array}$

\author{
Adoptar el programa de forma \\ progresiva a las necesidades de la \\ empresa.
}

El proyecto por realizar está basado en el sistema de planificación de recursos empresariales ya que este tipo de aplicaciones permiten hacerse cargo de distintas operaciones internas de una empresa, desde producción a distribución o incluso recursos humanos.

Dicho proyecto se pretende implementar ya que existe la necesidad de un sistema que permita unificar y definir la variedad de procesos que se encuentran en la empresa y de esta manera poder habilitar el flujo de datos entre ellos al recopilar la información entre todos sus componentes.

A través de ello, se pretende la optimización de los procesos empresariales, el fácil acceso a la información y crear diferentes módulos diseñados para cubrir las necesidades de cada departamento de la empresa, permitiendo crear un flujo de trabajo entre varios usuarios desde una base de datos centralizada.

La funcionalidad final del producto consta de los siguientes módulos: Acceso y Administración, Ingeniería, Almacén, Producción, Control de Calidad, Ventas y Embarques; siendo cada uno de ellos importantes para llevar a cabo el proceso de producción de esta empresa.

En este artículo se presenta el desarrollo de este sistema de información, describiendo la metodología utilizada para su realización, haciendo énfasis en el análisis de los procesos, así como también la estructura de la aplicación y en el último apartado se presentan las conclusiones de este proyecto, derivado de la construcción de esta plataforma web.

\section{Generalidades del Proyecto}

Zetrak S.A. de C.V. es una empresa grande y por ende cuenta con varios departamentos por lo que debe de adoptar tecnologías que la ayuden a generar más ventas, mejora de procesos y menos trabajo, todo esto en un solo sitio. 
Por ello, se pretende la implementación de un sistema que permita unificar y llevar al día la información detallada de las órdenes de trabajo y de producción que se generan en la empresa para que, de esta manera, las áreas descritas anteriormente puedan estar a la par con dicha información y así evitar el duplicado de ella ya que toda la información estará alojada en una base de datos centralizada y así poder evitar barreras de información entre las áreas, mejorando los procesos entre ellas.

Con la implementación de este sistema se pretende integrar los departamentos de la empresa, principalmente el área de ingeniería, laboratorio, embarques y ventas para el control de las órdenes de trabajo, puesto que, actualmente se tiene un sistema de información especializado para cada departamento, pero se pretende que con ello se pueda asegurar la existencia de una única base de datos donde se gestione toda esta información en tiempo real.

La página web tiene conexión a una base de datos que está estructurada en lenguaje MySQL.

Se usará la herramienta visual MySQL Workbench y el gestor de base de datos phpMyAdmin y al cierre de la captura de información, la base de datos se alojará en los servidores de phpMyAdmin de tal manera que los administradores podrán tener acceso a ella cuando así lo requieran.

Por medio de esta página web los usuarios pueden dar de alta órdenes de trabajo y los demás departamentos podrán coordinarse para darle seguimiento a dichas órdenes y de esta manera se suprimen las barreras de información entre las áreas y al contrario de ello, se integran todas entre sí, de forma tal que es mucho más fácil saber en qué está trabajando el resto y ver cómo puede uno ayudar o mejorar.

Por otra parte, permitirá la exportación en Excel con formato .xls de los registros existentes en cada apartado.

Este sistema está dirigido a los usuarios del departamento de ingeniería, que son los encargados de los diseños de los transformadores, área de aseguramiento de la calidad, facturación, embarques y ventas.
Todos ellos podrán manipular el sistema bajo un usuario y contraseña, pero dependiendo del área al que pertenezcan tendrán diferentes privilegios para capturar y consultar datos de las órdenes de trabajo.

La solución para optimizar el funcionamiento de la empresa y aumentar su rentabilidad final fue el desarrollo y uso del sistema llamado "Seguimiento de Órdenes de Producción", que es una solución de software de código abierto para la gestión de la producción y tiene como objetivo conseguir que se entreguen los productos pedidos, en este caso los transformadores, en las cantidades, fecha y calidad requerida.

Para lograr esto, se creó un flujo de trabajo que se convierta, con el tiempo, en algo característico de la empresa.

De esta manera se aporta la información en tiempo real de los materiales disponibles, de los productos, en resumen, de cada elemento necesario para planificar correctamente. $\mathrm{Y}$ a su vez, permite llevar a cabo un seguimiento ya que los datos recabados van conformando un retrato exhaustivo que indica si se cumplen los pasos establecidos en la planificación inicial.

Es una herramienta que, al estar integrada en todos los departamentos, es más sencillo tener una visión global del proceso productivo, desde que se da de alta la orden del producto, hasta la logística de almacenes $\mathrm{y}$ transporte.

Las principales funcionalidades de la aplicación son:

$\begin{array}{ll}- & \text { Gestión de órdenes abiertas } \\ \text { - } & \text { Gestión de órdenes cerradas } \\ \text { - } & \text { Control de las órdenes de trabajo } \\ \text { - } & \text { Alta de productos } \\ \text { - } & \text { Gestión de cobros y pagos } \\ \text { - } & \text { Reportes } \\ \text { - } & \text { Control de embarques }\end{array}$

El sistema pretende ser lo más eficiente posible, utilizando una interfaz gráfica simple, mostrando al inicio una pequeña galería de los principales tipos de transformadores que se fabrican en esta empresa, así como listados de las órdenes de trabajo dependiendo la sección a visualizar. 


\section{Metodología}

La metodología para el desarrollo de software que se utilizó fue en espiral debido a que es una metodología que se puede ir ajustando a los cambios que se presenten durante el desarrollo del software, es decir, nos permite retroceder a alguna etapa anterior para evaluar los riesgos y tratar de detectarlos para que al final no haya errores.

Una vez que se cumple un ciclo puedes volver a empezar utilizando el prototipo que acabas de crear y se vuelve un círculo vicioso que posiblemente no tenga final.

Esta metodología es utilizada ampliamente en la ingeniería del software, ya que hace uso de un enfoque evolutivo que permite al equipo de trabajo y al cliente entender las necesidades y reaccionar a los cambios que se detectan en cada iteración o espiral. Este modelo de desarrollo incorpora un elemento inexistente en otros modelos, denominado "análisis de riesgos" y que está definido por cuatro actividades principales que se describen en los cuadrantes de la figura 1.

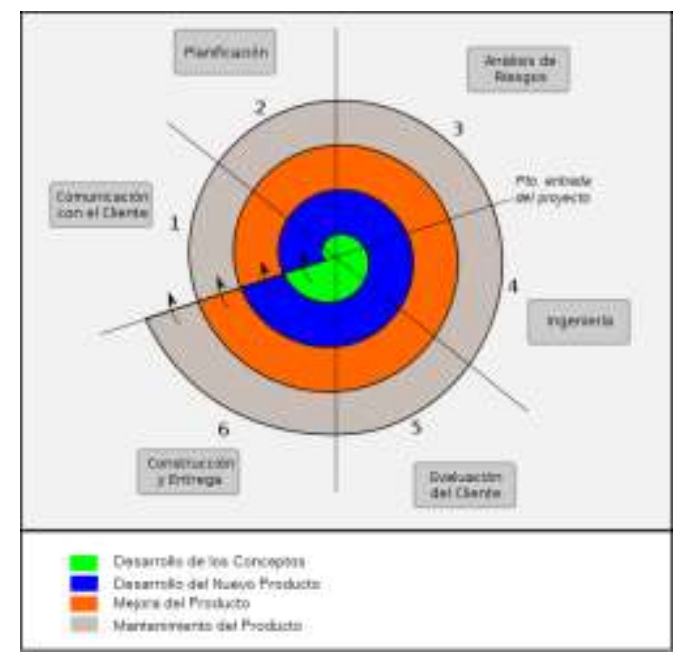

Figura 1 Modelo de desarrollo en Espiral Fuente: Ecured

Planificación: Se definen objetivos y restricciones, por lo que el equipo de desarrollo de software realizará actividades definidas en un circuito alrededor de la espiral. Es importante mencionar que cada paso en esta etapa da como resultado un ajuste del plan de proyecto.

Análisis de riesgo: Se identifican y se da solución a los riesgos, los cuales se consideran conforme se desarrolla cada iteración.
Ingeniería: Se desarrolla y verifica el producto del siguiente nivel, lo que da como resultado el desarrollo de una especificación del sistema, teniendo cada vez versiones más sofisticadas del sistema.

Evaluación del cliente: Se valoran los resultados y se hace la planificación para la siguiente fase. Es aquí cuando el costo y la programación de actividades se ajustan con base a los comentarios obtenidos por el cliente, y el líder del proyecto ajusta el número planeado de iteraciones que se requieren para concluir el sistema.

Durante cada una de las aplicaciones del modelo, se van generando versiones sucesivas del software, y cada iteración, permite obtener un modelo más completo del software, hasta llegar al sistema final que se pondrá en producción. Este modelo es considerado con un enfoque realista para el desarrollo de sistemas a gran escala, ya que, como el software evoluciona a medida que se va avanzando en el proceso, el desarrollador y el cliente reaccionan mejor ante los riesgos que pueda tener el sistema en cada interacción. (Grupo Espiral php, 2009)

\section{Herramientas para el desarrollo}

Para la programación se utilizó Netbeans IDE es un entorno de desarrollo integrado (IDE), modular, de base estándar (normalizado), escrito en el lenguaje de programación Java. El proyecto NetBeans consiste en un IDE de código abierto y una plataforma de aplicación, las cuales pueden ser usadas como una estructura de soporte general (framework) para compilar cualquier tipo de aplicación. (Netbeans, 2018)

Por otra parte, PHP es un lenguaje de código abierto muy popular especialmente adecuado para el desarrollo web y que puede ser incrustado en HTML.

PHP se caracteriza por ser un lenguaje gratuito y multiplataforma. Además de su posibilidad de acceso a muchos tipos de bases de datos, también es importante destacar su capacidad de crear páginas dinámicas, así como la posibilidad de separar el diseño del contenido de una web. (PHP, 2017) 
Servidor Apache es un software de servidor web gratuito y de código abierto con el cual se ejecutan el $46 \%$ de los sitios web de todo el mundo. El nombre oficial es Apache HTTP Server, y es mantenido y desarrollado por la Apache Software Foundation.

Les permite a los propietarios de sitios web servir contenido en la web, de ahí el nombre de "servidor web". (B., 2019)

En el almacenamiento de datos dentro del proyecto se ha escogido Maria DB ya que tiene una alta compatibilidad con MySQL ya que posee las mismas órdenes, interfaces, APIs y bibliotecas, siendo su objetivo poder cambiar un servidor por otro directamente $y$ es compatible con aplicaciones tan populares como WordPress, Drupal, etc. (Rodriguez, 2017)

La compatibilidad es tal que muchas veces el uso de MariaDB en lugar de MySQL es transparente para desarrolladores $\mathrm{O}$ administradores de sistemas. Prueba de ello es que, para arrancar los servicios de MariaDB, o para hacer login en el sistema gestor por medio de línea de comandos, se usan los mismos mecanismos ya conocidos en MySQL. (Blanch, y otros, 2018)

\section{Requisitos previos a la instalación}

Para instalar la página web en el servidor se deben de tener en cuenta estos requisitos:

- Un acceso al servidor de internet para instalar los archivos (FTP, SSH, etc.).

- $\quad$ Servidor Web Apache2 o superior.

- PHP4 como mínimo, con soporte de sesiones

- $\quad$ Acceso a una base de datos MySQL

- Disponer del módulo MySQL versión 4.6.4 o superior

Tener acceso a una base de datos MySQL para su activación. (Nombre de Usuario, Clave del Usuario, Nombre de la base de datos)

- Dirección IP del servidor de MySQL

\section{Resultados}

Como parte del ERP en funcionamiento se logró integarar 4 módulos que a continuación se detallan.

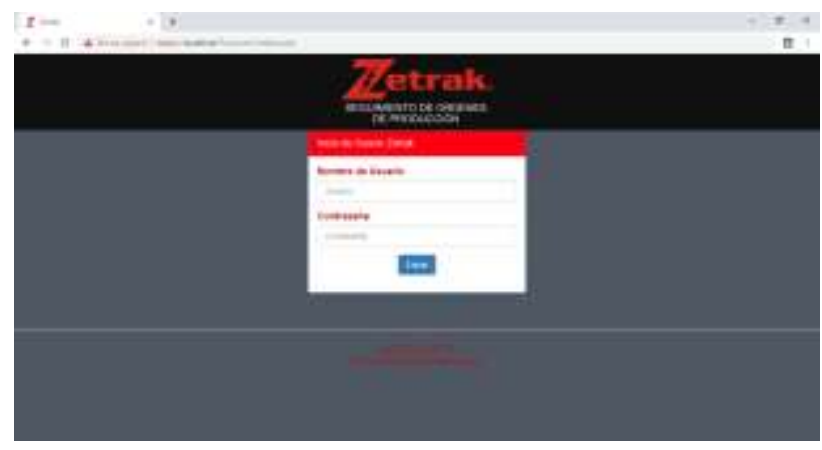

Figura 2 Inicio de sesión

Fuente: Elaboración Propia

Esta tarea permite loguear a los usuarios dados de alta en el sistema para que puedan usarlo.

Cuando se inicia sesión pide un usuario y una contraseña los cuales están asignados por el administrador.

Esta información la toma desde la base de datos. Si el usuario y/o contraseña son incorrectos no podrá ingresar a la pantalla principal.

\section{Pantalla de Bienvenida}

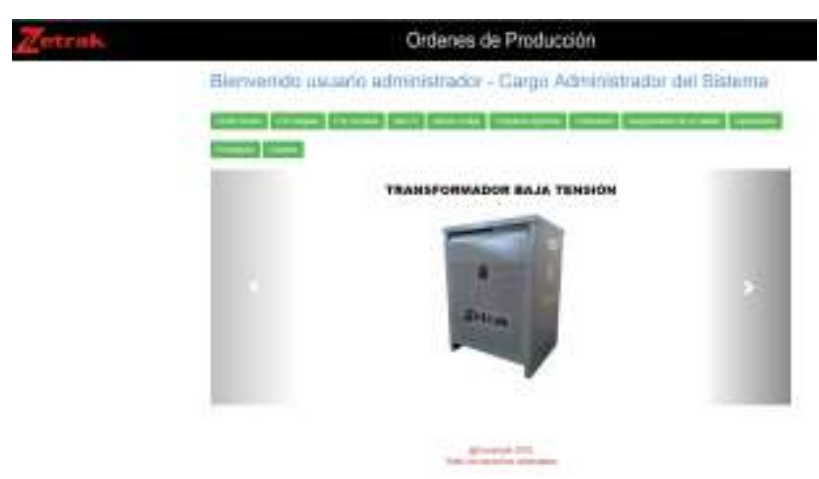

Figura 3 Bienvenida

Fuente: Elaboración Propia

En esta ventana se puede observar todo el menú disponible para el usuario con cargo de administrador y una galería tipo slider con los principales tipos de transformadores que se fabrican en la empresa.

\section{Módulo Ingeniería}

El botón "Programa Ingeniería" en su pantalla inicial muestra el listado de las órdenes de trabajo pendientes a realizar. En ese listado, los ingenieros o los encargados de realizar los diseños de los transformadores llevan a cabo un checklist de los diseños que aún faltan. 
A su vez, pueden realizar modificaciones sobre la información registrada.

La lista puede ser exportada en formato .xls para que los usuarios puedan manipular la información.

\section{Módulo Facturación}

Es en este apartado donde se encuentran los detalles financieros de los transformadores como el cliente, los pagos, los anticipos, la forma de pago, etc. La lista puede ser exportada en formato .xls

\section{Módulo Aseguramiento de Calidad}

En este apartado se encuentran las OT que no han sido liberadas por el departamento. De la misma manera, la lista puede ser exportada en formato .xls. Permite dar la liberación del transformador una vez que ya se hayan realizado las pruebas de laboratorio a los que son sometidos los transformadores para comprobar su calidad y eficiencia.

\section{Módulo de Embarques}

En este módulo se controla los detalles del tipo de transformador que se va a enviar, su destino, el chofer encargado del traslado, las fechas a entregar, etc.

En este apartado se integran las pruebas al software como parte de los resultados para asegurar la calidad del software,

Durante el desarrollo del sistema e implementación del sistema se llevaron a cabo las 3 pruebas de software al software para revisar cómo era el funcionamiento del sistema expuesto a diferentes ambientes.

Cada una de las pruebas consta de su objetivo general, breve descripción de la prueba y los resultados arrojados de cada prueba.

\section{Pruebas del sistema}

Objetivo: Asegura la apropiada navegación dentro del sistema, ingreso de datos, procesamiento, recuperación y la implementación apropiada de las reglas de negocios.
Este tipo de pruebas se basan en técnicas de caja negra, esto es, verificar el sistema (y sus procesos internos), la interacción con las aplicaciones que lo usan vía GUI y analizar las salidas o resultados.

Descripción de la prueba: Se ingresó la información correspondiente en la base de datos y después se revisó cómo esos datos eran extraídos a la interfaz del usuario y si así era cómo debían de extraerse los datos de la base.

Una vez revisado esto, se ingresaron datos desde la interfaz del usuario para comprobar la transferencia de los datos y ver si estos datos se almacenaban de manera correcta en la base.

Resultado: La entrada y salida de los datos fue la esperada, los datos se almacenan de manera correcta en la base y de igual forma son extraídos satisfactoriamente a la interfaz del usuario al momento de realizar la consulta.

\section{Pruebas de Carga}

Objetivo: Verificar el tiempo de respuesta del sistema para transacciones bajo diferentes condiciones de carga para medir la capacidad del sistema y continuar funcionando apropiadamente bajo diferentes condiciones. La meta de las pruebas de carga es determinar y asegurar que el sistema funciona apropiadamente aún más allá de la carga de trabajo máxima esperada. Adicionalmente, las pruebas de carga evalúan las características de desempeño (tiempos de respuesta, tasas de transacciones $\mathrm{y}$ otros aspectos sensibles al tiempo).

Descripción de la prueba: Cuando en el sistema se necesitaban consultar todas las OT existentes en la base de datos desde varios usuarios, se pudo percatar que sí registraba una demora de aproximadamente 30 segundos para realizar la consulta o extracción desde la base de datos.

Resultados: El sistema demostró demora en realizar diversas transacciones al mismo tiempo debido a que existen muchos registros en la base de datos y también porque el servidor en el que se encuentra alojado realizaba otros procesos como respaldo de archivos y ejecuta máquinas virtuales en segundo plano, aún así se consedra dentro de los rangos aceptados por los usuarios internos.

LEDESMA-URIBE, Norma Alejandra, LEDESMA-GARFIAS, Arisbeth y OLIVO-FLORES, Marco Antonio. Sistema ERP para el Seguimiento de Órdenes de Producción. Revista de Desarrollo Económico. 2019. 
3. Pruebas de seguridad y control de acceso

Objetivo: Verificar que un actor solo pueda acceder a las funciones y datos que su usuario tiene permitido y que solo los actores con acceso al sistema y a la aplicación están habilitados para accederla.

Descripción de la prueba: Se intentó ingresar al sistema con datos incorrectos a los que se encuentran almacenados en la base de datos y el resultado fue le esperado porque no dejó ingresar al sistema.

Se verificó que los usuarios que se encuentran dados de alta en la base de datos pudieran ingresar al sistema de acuerdo con el cargo que poseen, es decir, con los permisos acordados al momento de crearlos.

Resultados: El sistema negó la entrada al sistema de usuarios no existentes en la base de datos, así como también de algún usuario y/o contraseña incorrectos.

Con los resultados de las pruebas favorables para el sistema se implantó en el servidor de producción y se encidentra en operación en las dos plantas del país, ahora se desarrollan 5 módulos más para integración de otras áreas como compras y RH.

\section{Conclusiones}

Resulta de gran importancia utilizar las tecnologías de la información para contribuir en un sector tan importante, con el presente trabajo desarrollo del ERP para el seguimiento de las órdenes de producción, de la empresa Zetrak S.A. de C.V., se demuestra que un sistema con esta funcionalidad trae como beneficios en primer plano en la parte económica y pero sobre todo agiliza el monitoreo que permite la solución en un menor tiempo y recursos involucrados en cada una de sus órdenes de trabajo solicitadas a la empresa.

Con los servicios web se ha incrementado el uso de ERP en pequeñas y medianas empresas, a las que en años anteriores por el tipo de plataforma se encontraban los beneficios, pero los costos las volvían inaccesibles a las Pymes.
Este sistema ha sido aceptado favorablemente por los usuarios propios de cada área, ya que encuentran los beneficios de tener en un solo lugar la información propia de cada una de las órdenes de producción y la información de embarques y los componentes que autoriza el área de ingeniería.

\section{Referencias}

Acosta, O., \& del Carmen, J. (2019). Propuesta de mejora del proceso en un centro de producción de hilo dental.

B., G. (18 de Enero de 2019). Hostinger. Recuperado el 01 de Marzo de 2019, de https://www.hostinger.mx/tutoriales/que-esapache/

Blanch, A., Galán, H. G., León, M., Ruesgas, M. G., Arias, S., \& García, J. B. (31 de Enero de 2018). Arsys. Recuperado el 01 de Marzo de 2019, de https://www.arsys.es/blog/programa cion/cuando-utilizar-mariadb/

Cuetter, D. M. (24 de Enero de 2017). Inteligencia de Negocios. Recuperado el 10 de Mayo de 2019, de https://revistaempresarial.com/tecnologia/inteli gencia-de-negocios/erp-clave-mejorarproductividad-empresas/

Delgado, C., Milagros, C. F., \& Dill'erva Monroe, M. G. (2019). Desarrollo de un modelo de gestión de planeamiento y control de producción para las Mypes del sector hotelero de 2 y 3 estrellas de Lima Metropolitana.

Dictionary. (20 de 09 de 2018). Obtenido de Dictionary:

https://es.thefreedictionary.com/bast\%C3\%B3n

Gálvez Ocaña, K. C., \& Sigüenza Abanto, J. C. (2019). Planeamiento Estratégico de Sistemas de Información para mejorar la Gestión Administrativa de la Empresa Construcciones y Servicios Metálicos SAC".

Grupo Espiral php. (08 de Agosto de 2009). Modelo espiral. Recuperado el 10 de Mayo de 2019, de http://modeloespiral.blogspot.com/

Netbeans. (2018). Netbeans. Recuperado el 01 de Marzo de 2019, de https://netbeans.org/community/releases/61/ind ex_es.html 
Oracle. (2019). Recuperado el 28 de Febrero de 2019 , de https://www.oracle.com/mx/applications/erp/w hat-is-erp.html

PHP. (2017). Recuperado el 01 de 03 de 2019, de http://php.net/manual/es/intro-whatis.php

Rodriguez, A. (29 de Agosto de 2017). EcuRed. Recuperado el 01 de Marzo de 2019, de https://www.ecured.cu/MariaDB

Rojas, R., \& Cleyder, G. (2019). Mejora de procesos para optimizar el desarrollo de muestras de prendas de vestir en una empresa de confecciones.

Sotomayor Zumarán, A., Idrugo, G., \& Diego, J. (2019). Influencia de la planificación y control en el desempeño operacional del área de producción en Mypes de calzados en Lima Metropolitana 2019.

TIC portal. (2019). Recuperado el 28 de Febrero de 2019, de https://www.ticportal.es/temas/enterpriseresource-planning/que-es-sistema-erp 